\title{
Geraniol Attenuates Oxidative Stress and Neuro- inflammation Mediated Cognitive Impairment in D-galactose-induced Mouse Aging Model
}

Peramaiyan Rajendran ( $\sim$ prajendran@kfu.edu.sa )

King Faisal University

Rebai Ben Ammar

King Faisal University

Fatma J Al-Saeedi

Kuwait University

Saeed Y. AlRamadan

King Faisal University

Mohammad Bani Ismail

King Faisal University

Vishnu Priya Veeraraghavan

Saveetha University

Sarah Abdulaziz Alamer

King Faisal University

Nasir Alawwad

Al Baha University

Mohammed Salem Moqbel

King Faisal University

Emad A Ahmed

King Faisal University

\section{Research Article}

Keywords: D-galactose, Geraniol, Cognitive disorder, Nrf2, Apoptosis

Posted Date: January 11th, 2022

DOI: https://doi.org/10.21203/rs.3.rs-1215880/v1

License: (c) (1) This work is licensed under a Creative Commons Attribution 4.0 International License.

Read Full License 


\section{Abstract}

D-galactose (D-gal) is a reducing sugar drug can induce artificial senescence and aging process that mimic natural aging along with the accompanying brain and liver injury in experimental animals. Therefore, chronic D-gal administration is widely used to induce cognitive impairment, Alzheimer disease and aging in rodents' models. Aging is a phenomenon in which oxidative stress and apoptosis play a vital role. Geraniol (GNL) belongs to the acyclic isoprenoid monoterpenes, presents in essential oils such as those from Cinnamomum tenuipilum and Valeriana officinalis. In the present study, we examined the effects of GNL on D-gal-induced oxidative stress and neuro-inflammation mediated memory loss in mice. Analyzing the behavioral differences between control and treated groups, including the elderly mice, revealed that GNL significantly improved memory in mice treated with D-gal-induced memory loss (supplementary videos are provided). The anti-inflammatory and the anti-oxidative role of GNL were confirmed by both histopathological investigations and biochemical analyses. Mechanistically, GNL appears to activate PI3K/Akt and thus upregulates the nuclear factor erythroid 2-related factor 2 (Nrf2) and the heme oxygenase 1 (HO-1) to reduce the oxidative stress and apoptosis induced after D-gal treatment leading to easing of neurological deficits and cognitive dysfunction in D-gal-induced aging mouse models. Accordingly, our comprehensive behavioral analysis and bioassays suggest GNL as a promising agent preventing cognitive impairment and neurological deficits associated with aging.

\section{Introduction}

Chronic oxidative stress is a major factor in neurodegenerative diseases and aging due to the accumulation of reactive oxygen species ${ }^{1}$. Aging is a natural phenomenon associated with an increase in reactive oxygen species, and then a decline in antioxidant defense mechanisms, leading to increased risk of diseases such as Alzheimer, cancer and heart diseases ${ }^{2}$. The decline of brain function and cognitive impairment by aging are attributed to oxidative stress, DNA damage and then mutations induction that cause abnormal protein aggregation, neuro-inflammation and neurodegeneration ${ }^{34}$. .

Several recent studies have clarified that D-gal injection could induce brain aging through increasing oxidative damage, inflammation and apoptosis, as well as lowering brain function leading to cognitive impairment ${ }^{6-9}$. In fact, our body can synthesize D-gal, which is a reducing sugar constitutes a part of glycolipids and glycoproteins, obtained from a variety of foods. The in vivo accumulation of D-gal may result in the formation of advanced glycation end products (AGEs). D-gal can directly produce ROS, or indirectly triggers free radical production, which results in oxidative stress and then apoptosis ${ }^{8,9}$. On the other hand, cells have protective mechanisms and antioxidant system protects cells from oxidative and proteotoxic stress. One of these molecules is the nuclear factor (erythroid-derived-2)-like 2 (Nrf2). The antioxidant response element (ARE) is responsible for inducing NRF2 genes. The function of Nrf2 is to keep cells from being damaged by oxidative stress induced by reactive oxygen or nitrogen species ${ }^{10-12}$. In the brain, Nrf2 and related pathways help to combat oxidative stress, making them appealing targets for attacking cognitive decline. In this line, several recent studies have documented that with aging, the 
decline in cognitive abilities is associated with a decrease in Nrf2 expression level. Therefore, the Nrf2 pathway is a promising target for treating neurodegenerative diseases ${ }^{13-16}$. In this line, to regulate oxidative stress and inflammatory response, Nrf2 was found to be a key factor of the phosphoinositide 3kinase/ protein kinase B, PKB (PI3K/Akt) downstream molecules ${ }^{17}$. In addition, D-gal-induced aging was reported to inhibit the PI3K/Akt pathway and inactivate Nrf2-mediated antioxidants expression in liver and brain ${ }^{18,19}$.

Geraniol (GNL) is one of the representatives of acyclic isoprenoid monoterpenes, which can be extracted from the aromatic plants' essential oils such as Cinnamomum tenuipilum and Valeriana officinalis, and other plants ${ }^{20}$. Researchers found that GNL has a variety of pharmacological effects, including, antiinflammatory, antitumor, anti-oxidative and antimicrobial activities ${ }^{21-23}$.

In the present study, GNL was tested for its effects on oxidative stress and neuro-inflammation mediated cognitive impairment in dgalinduced aged mice model. Mechanistic action of GNL on Nrf2 and AKT signaling pathways have been explored. Biochemical and histopathological data reveled a neuroprotective effect of GNL on aged mice model. This has been clearly confirmed through evaluating behavioral analysis (videos are supplemented) based on the spatial learning and memory loss in aged model and GNL treated aged model relative to the control mice and old mice.

\section{Materials Methods}

\subsection{Chemicals}

Geraniol and D-gal was purchased from Sigma Aldrich in Germany. Antibodies against HO-1 (\# PA577833), PARP (PA5-16452), pAkt (\# 44-621G), NQ01 (\# PA5-82294), pPI3K (\#PA5-104853), BCL2 (\# PA527094), PI3K (\# PA5-29220), Nrf2 (\# PA5-105664), ß-actin (\# PA5-78716) and Akt (\# 44-609G) were sourced from Invitrogen; Thermo Fisher Scientific, Inc. (Waltham, MA, USA). Anti-cleaved caspase-3 (ab32042) antibody was purchased from Abcam (Branford, CT, USA). MDA, SOD, GPx, and CAT were measured using commercial kits (from Cayman Chemical, Ann Arbor, MI, USA) where experiments were done according to the manufactured protocol.

\subsection{Animals and Treatments}

Thirty-six albino male mice weighing 25-30 gm were purchased from Charles River Laboratories (Écully, France) provided. All methods were carried out in accordance with relevant guidelines and according to regulations of King Faisal University. All experimental protocols were approved by King Faisal University Research Ethics Committee (KFU-REC/2021-01-15) before starting experiments and then the informed consent was obtained according to legal guardians. In a normal lab atmosphere, the mice were kept at a temperature of $22 \pm 2^{\circ} \mathrm{C}$ with a $12 / 12 \mathrm{~h}$ light/dark cycle. Six groups (6 mice each) were randomly selected. While mice of the control group received equal amounts of physiological saline once daily for 9 weeks, the 2nd group (D-gal control, II) was given orally D-gal ( $150 \mathrm{mg} / \mathrm{wt})$ dissolved in normal saline solution 
$(0.9 \%, w / v)$ for 9 weeks. Mice of the third group (III) were treated with GNL (40 mg/kg wt) alone in the first week and starting from the second week up till the 10th week, mice were treated with oral gavage (without anesthesia) with D-gal (150 mg/wt) and with GNL (50 mg/kg wt) $4 \mathrm{~h}$ later. Mice of the fourth group (the drug control group, IV), were treated with GNL from the second week up till the end of the experiment. In addition, 6 mice of each sex were used as two untreated groups at 4 months (group 5) and 16 months (group 6) of age to analyze the cognitive impairment differences between mature and elderly mice. This was also done to determine whether treatment prevented or reversed the kinetics of cognitive decline. Mice were sedated with ether and sacrificed at the end of the experiment. To measure proinflammatory cytokines and biochemical markers, blood was collected via cardiac puncture and serum was separated and stored at $-80^{\circ} \mathrm{C}$. Using a sensitive balance (Nimbus, MK, UK), brains and spleen were

excised and weighed before being fixed or for histopathological analysis or homogenized for biochemical investigations. For biochemical evaluation, the brain hippocampus was homogenized in $0.1 \mathrm{M} \mathrm{Tris-HCl}$ buffer (pH 7.4).

\subsection{Behavioral Analysis}

Next, we used different testes including Morris' water maze test, Y-maze test and object-place recognition and open-field test to study the behavior of control and treated mice ( $n=6 /$ group). 2.4 Morris' Water Maze Test Morris' water maze test was used to estimate the spatial learning and memory after GNL and D-gal treatment. A circular tank has a diameter of 100 centimeters and a height of 20 centimeters was used. A white ink to the water $\left(23 \pm 1^{\circ} \mathrm{C}\right)$ to make the tank opaque. A small platform was submerged $12 \mathrm{~cm}$ below the surface of water at the center of the third quadrant. MWM entailed a navigation training trial and a probe test. Each mouse was subjected to four tests per day for 4 days straight as part of the learning and memory training. Whenever the mice couldn't find the platform in 45 seconds, they were artificially guided to it and stayed there for 15 seconds to memorize it. The probe test without the platform was conducted for 45 seconds in the tank after the training trial.

\subsection{Y-Maze Test}

Studies have shown that excessive spontaneous alteration behavior is linked to enhanced cognitive performance. Then, a Y-maze made of brown pointed sheet with three arms that were $60 \mathrm{~cm} l o n g, 15 \mathrm{~cm}$ high, and $15 \mathrm{~cm}$ wide at the bottom and top was used to analyze mice behavior. Three 5-minute sessions were given to the mice at the center. In the arms of the $Y$, theirs food. The mouse capability to remember if food is located in one arm or the other. By this way conditions could affect learning and memory and help to analyze the mice behavior. For the food identification, each mouse's arm position was recorded manually 3 times in different days for 5 minutes each.

\subsection{Object Place Recognition}

Based on previous studies, we modified a standard object recognition task to test the recognition of shortterm object-place. ${ }^{24}$. Through the experiment, each mouse was allowed to adapt for 10 min in an open box (120X $80 \mathrm{~cm})$. Then they began training at 30-min intervals. Each mouse was given 5 minutes to practice object-place recognition in a quadratic box (150X100 cm W, $25 \mathrm{~cm} \mathrm{H})$. In the training session, 
two identical plastic objects were positioned diagonally $120 \mathrm{~cm}$ from the wall in the apparatus. Between the training and testing, there was a one-day rest period. Two identical objects were placed $120 \mathrm{~cm}$ from the wall and the mice during the test. Animals' behavior was evaluated by how much time they spent looking at each object.

\subsection{Cytokine measurements}

ELISA kits were used to measure TNF- $a(\mathrm{pg} / \mathrm{mL}), \mathrm{IL}-1 \beta(\mathrm{pg} / \mathrm{mL})$, and IL- $6(\mathrm{pg} / \mathrm{mL})$ in a serum sample. ( MC3011 for TNF-a; BMS6002 IL- $\beta 1$; KMC0061 for IL-6) obtained from Invitrogen (USA) ${ }^{25}$.

\subsection{Protein Extraction from brain hippocampus}

Homogenized hippocampus tissues were centrifuged at 13,000 r.p.m. at $4^{\circ} \mathrm{C}$ for 25 minutes with a cocktail of phosphatase and protease inhibitors. Supernatants of all samples were stored at $-80^{\circ} \mathrm{C}$ to be used for biochemical investigations.

\subsection{Biochemical Analysis}

Spectrophotometry was used to measure CAT (U/mg protein), SOD (U/mg protein), MDA ( $\mathrm{nmol} / \mathrm{mg}$ protein) and GPX (U/mg protein) in the serum and hippocampus tissue. MDA, GSH, SOD, CAT and Gpx assay kits (Cayman Chemical, Ann Arbor, MI, USA) were utilized for analysis ${ }^{25}$.

\subsection{AChE Evaluation}

Using Elabscience Biotechnology Inc.'s (Houston, TX, USA), we measured the levels of AChE in the hippocampal homogenates using a quantitative sandwich ELISA.

\subsection{Histopathological Evaluations}

We collected brain, liver and spleen from mice, fixed them in $4 \%$ polyformaldehyde at $4^{\circ} \mathrm{C}$ for $4 \mathrm{~h}$, soaked them overnight in $100 \mathrm{mM}$ sodium phosphate buffer (containing 30\% sucrose), and embedded them in paraffin. After that, sections were stained with haematoxylin and eosin (H\&E) and Nissl staining to be investigated under microscope.

\subsection{Western Blot Analysis}

RIPA lysis buffer was used to extract proteins from brain hippocampus tissue homogenates. Using a BioRad protein assay, and bovine serum albumin (BSA) as a reference standard, tissue homogenates were collected by centrifugation at $12,000 \mathrm{rpm}$ for $30 \mathrm{~min}$ at $4^{\circ} \mathrm{C}$. We isolated equal amounts of protein $(40 * \mathrm{~g})$ on $10 \%$ sodium dodecyl sulfate-polyacrylamide gel electrophoresis (SDS-PAGE) and then transferred it to PVDF membranes. Block it with 5\% nonfat dry milk in Tris-buffered saline with Tween 20 (TBST, 150 $\mathrm{mM} \mathrm{NaCl}, 20 \mathrm{mM}$ Tris- $\mathrm{HCl}$, and $0.1 \%$ Tween 20) for 1 hour at room temperature. IgG labeled goat antirabbit lgG (1:2000) or anti-mouse $\lg G(1: 4000)$ was used as a secondary antibody after membranes were 
washed three times with TBST. Incubation time was 1.5 hours. The samples were examined with a LI-COR chemiluminescence imaging system (3600-00-C-Digit Blot Scanner). Graphs of the densitometric band intensities were generated and analyzed using LI-COR Biosciences Image Studio Lite software (Lincoln, $\mathrm{NE}, \mathrm{USA}$ ) normalized to the untreated control band, which was set to 1 .

\section{Results}

\subsection{Effect of GNL on body weight and organ index}

As shown in Fig. 1B and after week 10 of treatment, the d-gal model group had a significant decrease on body weight relative to the control group $(p<0.05)$, however the other administrated groups reversed $d-$ gal-induced weight loss $(\mathrm{p}<0.05)$. In the meantime, brain index, liver $(\mathrm{mg} / \mathrm{g})$ index of the $\mathrm{d}$-gal group were decreased significantly compared with the control group $(p<0.05)$. However, the administration of GNL $(40 \mathrm{mg} / \mathrm{kg})$ significantly attenuated the weight loss and increased spleen indexes in comparison to the D-gal group. In group V and group VI no significant variation was found. These results indicated that GNL modulated the reduction of body weight and the decrease in brain and liver indexes in D-gal model.

\subsection{Effect of GNL on mice behavior}

\subsubsection{Morris water maze}

The effects of GNL on spatial memory and learning were studied using the Morris water maze ${ }^{26}$. Chronic D-gal administration resulted in a significant longer time for mice to reach the platform $(P<0.05)$, while GNL therapy induced a significant reduction in escape latencies (Fig. $2 A)$ (Video $S 1)(P<0.05)$. On the other hand, D-gal-treated aging mice spent significantly less time in the goal area than the control group in the spatial probe trial (Video S1) $(P<0.05)$. In these poor conditions, mice in the D-gal plus GNL group showed remarkable reversion. Additionally, the aging mimic mice in the model group displayed similar behavior to the group VI. On the other hand, aged animals have a longer latency to reach the platform compared with young animals under 4 months (Group IV). These findings demonstrated that GNL could restore the $\mathrm{D}$-gal-induced deficit in memory and learning abilities.

\subsubsection{Y-maze task}

Using the Y-maze task, we analyzed spatial working memory using spontaneous alteration. D-gal treated mice were less likely to enter food rewards placed around its arms, which indicates no exploratory behavior. A significant increase in spontaneously altered behavior was observed in mice which received GNL either alone or in combination with $D$-gal $(P<0.05)$, indicating that GNL improved memory in D-galtreated mice (Fig. 2B) (Video S2). Thus, GNL may improve working memory. It doesn't appear that there are any significant differences between groups III (Figure 2B) and control group in behavior to enter food rewards placed arms (Figure 2B). In addition, compared to 4-month-old young animals, animals in group $\mathrm{VI}$ are significantly less likely to enter the food rewards arm. Therefore, GNL appears to attenuate D-galinduced ageing in mice model. 


\subsubsection{GNL improves the D-gal-induced decline in object recognition}

We explored the effect of treating with GNL on novel object recognition (NOR). When comparing the NOR test to the training session, a noticeable increase in object recognition index was observed in the control group. D-gal treatment also exacerbated the amnesic effect (Figure 2C) (Video S3), while pretreatment with GNL $(40 \mathrm{mg} / \mathrm{kg})(\mathrm{p}<0.05)$ ameliorated it (Video S3). The long-term exposure to D-gal can lead to a recognition deficit in the NOR test, but you can improve it by pre-treatment with GNL. GNL could improve the memory impairment results in by D-gal treatment. The young animals in Group $\mathrm{V}$ moved quickly and used active recognition, while the old animals moved slowly, often stood still, and didn't explore.

\subsection{Effects of GNL on Acetylcholinesterase (AChE) activity in aged mice model}

Acetylcholinesterase (AChE) affects learning andmemory and controls cholinergic synapses and its activity, and therefore is a reliable indicator of cholinergic activity ${ }^{27}$. As indicated in Fig. 2D the AChE activity in the D-gal group is significantly higher than in the control group. Following supplementation with GNL, the levels of AChE in aging mice induced by D-gal were markedly reduced compared to the GNL group with D-gal. In group $\mathrm{VI}$, the levels were significantly higher than those in group $\mathrm{V}$. This proved that GNL can improve cognitive decline through modulating the level of AChE that has been dysregulated after D-gal treatment.

\subsection{Effect of GNL on pro-inflammatory cytokines induced by D-gal}

In here, the D-gal treated mice overexpress inflammatory cytokines, which is in line with previous reports 28,29 . We found that pro-inflammatory cytokines such as TNF-a, IL-6, and IL-1 $\beta$ were about two times higher in the D-gal-treated group than in the control group. However, in D-gal treated group, serum levels of TNF-a, IL-6, and IL-1 $\beta$ were lower than D-gal model group after GNL administration $(P<0.05)$. This indicated an anti-inflammatory role for GNL on D-gal induced immune defect on mice.

\subsection{Effect of GNL on antioxidants level and activities of MDA induced by D-gal}

Increasing evidence indicated that oxidative stress is connected to the biology of aging ${ }^{30,31}$. Therefore, we investigated several antioxidants that could scavenge ROS in the serum and hippocampus of D-gal induced mice, including SOD, CAT, and GPX. According to Figs. $3 A$ and $B$, there was a noticeable decrease in SOD, CAT, and GPx levels after D-gal exposure compared to the vehicle group $(P<0.05)$. However, in serum and hippocampal, GNL treatment reversed the decline due to its antioxidant activity. Group IV mice treated with GNL alone showed no significant difference in serum and hippocampus CAT, GSH-Px, and SOD. GNL treatment enhanced a better antioxidant activity in the hippocampus, according to our results. 
On the other hand, MDA levels in serum and hippocampus homogenates significantly increased in the Dgal group, whereas GNL treatment at $40 \mathrm{mg} / \mathrm{wt}$ significantly abated the rise in serum and hippocampus MDA levels (Fig. 3A and B). In addition, as seen in Figure $3 A$ and B, the MDA levels of aged mice (Group $\mathrm{VI})$ are much higher than those of young control mice $(\mathrm{V})$. Together these data revealed a protective effect of GNL on D-gal induced oxidative stress in Brain and serum of mice.

\subsection{Effect of GNL on D-gal induced changes in the expression of PI3K/Akt}

Next, we measured AKT and PI3K expression levels in control and treated groups. Although total PI3K and AKT protein levels were similar among all groups, D-gal exposure prevented PI3K signaling, as reflected by the reduced expression of pPI3K and pAKT expression. This inhibition of pPI3K and pAKT by D-gal was significantly alleviated by GNL treatment (Fig. 4A). In 4-month-old animals (Fig. 4B), the expression of PI3K and AKT showed insignificant variation relative to group I, but in group VI (old mice), the level of phosphorylated of PI3K/AKT showed significant decrease.

\subsection{Effects of GNL on Nrf2, HO-1 and NQO-1 expressions in mouse hippocampus}

In agreement with the inhibition of PI3K, the expression of Nrf2, $\mathrm{HO}-1$ and NQO-1 was reduced in GNL with D-gal treated mice (Fig. 5a) compared to that of D-gal model mice. Further, Nrf2, HO-1, and NQO-1 expression in natural aging mice (Fig. 5a) decreased in similar way to D-gal induced aging in mice. In group III (D-gal with GNL) treated mice activation of this protein was significantly elevated $(p<0.05)$. However, GNL alone, vehicle control and young (4 months old) weren't significantly different (Fig. 5b).

\subsection{GNL on expression of RAGE and BACE-1 Proteins}

The D-gal was reported to increase RAGE protein levels, which stimulates hippocampus cells in neuroinflammatory neurodegeneration ${ }^{32,33}$. To analyze GNL's effect on RAGE and BACE-1, we conducted western blots analysis using protein extract from all experimental groups. The immunoblots showed that D-gal alone significantly increased RAGE and BACE-1 protein expression levels in hippocampus regions in comparison with the control group. However, co-administration of GNL + D-gal markedly reduced RAGE, and BACE-1 expression (Figure 6A). Actually, the immunoblot results showed that GNL is not toxic to normal mice brains hippocampus because no significant difference was found between normal control mice and mice treated with GNL by itself (Figure 6A). RAGE and BACE-1 expression were also up in natural aging mice (16 month old) (Fig. 6b), similar to D-gal induced aging mice. In contrast, GNL alone, vehicle control and young (4 months old) had no effect (Fig. 6b).

\subsection{GNL supplementation inhibited D-gal-induced hippocampal apoptosis}

D-gal induced aging was tied to hippocampal apoptosis, according to accumulating evidence ${ }^{34,35}$. Here, we performed western blot to check the ability of GNL supplementation to inhibit apoptosis. As shown in 
Fig. 7A and $\mathrm{B}$, the expression of caspase-3 and $\mathrm{Bax} / \mathrm{Bcl}-2$ are significantly higher after $\mathrm{D}$-gal treatment compared to the control group. By supplementing $\mathrm{GNL}$, the upregulation of these apoptosis-related proteins was dramatically attenuated. Natural aging mice also showed an increase in the Bax/Bcl-2 ratio (Fig. 7b). However, GNL alone, vehicle control and young (4 months old) had no significant effect (Fig. 7a and $b$ ). This revealed a protective role for GNL on D-gal inducing hippocampal apoptosis.

\subsection{GNL on Ameliorated Histopathological Alterations of the Brain, Liver and Spleen}

Hippocampus of brain (regions CA1 and CA3) of treated mice was stained with H\&E (Fig. 8) to investigate the pathological changes relative to the control. As indicated, D-gal causes neurodegeneration characterized by focal disappearance and a decrease in density, as well as a necrosis represented by shrunken neurons displaying dark nuclear staining (arrows) relative to the control group. In the meantime, in group III (6 weeks), GNL showed a protective effect where mild neurodegeneration was seen (thin arrows) with highly pronounced normal neurons (thick arrow). Both of control group and group IV (the drug control) showed normal appearance of neurons shown in G4 (6 weeks). However, in group V (4 months), mild decrease in neuronal density was seen relative to the control. On the other hand, severe neural loss, degeneration, and necrosis (arrows) were seen in old mice (16 months). In the same line, Dgal induced histopathological alteration in liver and spleen (supplementary data), these changes have been modulated by GNL treatment. Together these data support protective effects on GNL at the histological level that in synchronization with what we have seen using behavioral and biochemical analysis.

\section{Discussion}

Life expectancy in the 21st century is rising, resulting in more age-related illnesses, such as memory impairment and Alzheimer's disease. Herein, GNL was studied for its protective effect on D-galactoseinduced aging in mice. We found that GNL was able to improve spatial location learning and to reduce the extent of memory impairment (please see suppl videos) as well as it protected neurons in the hippocampus from oxidative damage and apoptosis induced by D-galactose. Mechanistically, to alleviate D-gal effect on brain, GNL appears to activate Nrf2, the key factor among PI3K/Akt downstream through modulating the phosphorylation of PI3K/Akt. Therefore, GNL activates Nrf2 via the PI3K/AKT pathway to enhance the activity of antioxidant enzymes, like HO-1, NQO-1, and SOD.

The effect of GNL on memory impairment and learning in the D-galactose-induced aging model is demonstrated here for the 1st time. Interestingly, consistent with our results, GNL was reported to enhance a neuro-protective effect against ischemic injury in the brain and to pass efficiently through the blood-brain barrier ${ }^{36,37}$. In the present study, we provide robust evidence (suppl videos) to show that GNL is a potential drug in can modulate behavior changes induced in mice after D-gal treatment. By applying the Y-maze and open field tests, we found significant variations between the vehicle and D-galtreated mice, suggesting that D-gal injection causes motor abnormalities that led to impairments of 
novelty-induced exploratory behavior. Furthermore, our data showed that treating mice with GNL for 8 weeks can reverse the D-gal-induced behavioral changes and then GNL can protect against D-gal-induced memory loss and spatial learning impairment. In consistent with our results, recently, GNL was suggested as a promising therapeutic agent in improving cognitive function and neurotoxicity induced in rats by ZnO-NPson, behavioral and biochemical evidence were provided ${ }^{38}$.

Several age-associated characteristics were found to be related to the increase in reactive oxygen species, and thus leading to the induction of oxidative stress and inflammation 39,40 . Interestingly, in the present study, both aging model and old mice displayed these characteristics including oxidative damage, apoptosis, and induction inflammatory response. The increase in ROS can cause NF-KB to move rapidly from the cytosol to the nucleus, resulting in an increase in inflammatory cytokines such as TNF- $a, \mathrm{IL}-6$, and IL-1 $\beta .{ }^{3}$. In this line, when we gave mice D-gal for 9 weeks orally, the serum concentrations of TNF-a, IL-1 $\beta$, and IL-6 were markedly increased in model animal. However, GNL substantially inhibited inflammatory cytokine production, and hence GNL was able to attenuate the induced inflammatory response in D-gal-treated mice. In addition, D-gal attenuated brain hippocampus antioxidant enzymes; SOD, CAT, and GPX in mice serum and hippocampi. to the exhaustion of antioxidant enzymes is due to the increase of ROS production with aging explaining the induction of age-related degenerative disease by D-gal ${ }^{41}$. However, the increase of brain hippocampus and serum SOD, CAT, and GPx activities in D-gal treated mice are in-line with several previous studies that indicated the antioxidant properties of GNL. For instance, Lin and colleagues studied the effect of GNL on methicillin-resistant Staphylococcus aureus (MRSA) infections in mice where GNL increased the antioxidant activity of SOD, and reduced ROS and MDA in the kidneys ${ }^{42}$. In addition, Farokhcheh et al. Showed that GNL reduced ROS in neurotoxicity cells in brain tissue ${ }^{38}$. As a result, this implies that the effect of GNL on aging may be mediated by modulating the imbalance between free radicals and antioxidants.

$\mathrm{PI}$ KK/Akt signaling is considered as an important upstream pathway regulating Nrf2 ${ }^{43}, 44$. Activation of $\mathrm{PI} 3 \mathrm{~K} /$ Akt signaling pathway promotes the dissociation downstream molecules including Nrf2 and Keap1, thereby regulating the nuclear expression of Nrf2 ${ }^{45,46}$. Numerous studies hinted that the PI3K/Akt signaling pathway protects against D-gal-induced brain injury by modulating Nrf2 ${ }^{46,47}$. Additionally, several natural compounds were found to improve the behavioral dysfunction and neurological deficits in D-galactose-induced aging in mice via activation of PI3K/Akt/Nrf2 pathway ${ }^{48-50}$. Results of the current work demonstrated that phosphorylated levels of PI3K and Akt are significantly upregulated after GNL treatment, which revealed that GNL can activate PI3K/Akt signaling pathway to promote Nrf2 nuclear translocation via reduction D-galactose-induced oxidative stress. Neurodegenerative diseases are treated by targeting Nrf2 pathway ${ }^{51}$. Keap1, a cysteine-rich protein present in the cytoplasm, binds to Nrf2 under normal conditions. However, when Keap1 denatures, Nrf2 translocate into the nucleus, binds to antioxidant elements (ARE), and turns on antioxidant enzyme genes, like SOD and CAT ${ }^{52}$. Studies have shown that aged animals have less nuclear translocation of $\mathrm{Nrf2}{ }^{45}$. This model showed similar results to 
previous studies where D-galactose treatment inhibited Nrf2 translocation to the nucleus ${ }^{48}$, however, treatment the model with GNL have shown good results.

The neuroprotective effect can be attributed to NQO-1 and HO-1, antioxidant enzymes ${ }^{53}$. In aged model treated with GNL, the accumulation nuclear factor Nrf2, increased expression of NQO-1 and HO-1, the downstream proteins of Nrf2, which confirms that Nrf2 is involved, but also demonstrates how critical they are for protecting GNL from free radical damage. Rodent D-gal brain neurotoxicity is also attributed to oxidative stress-induced apoptosis ${ }^{46}$. Herein, brain hippocampus of mice treated with D-gal showed elevated level of Bax and caspase-3, and lowere expression of Bcl-2 ${ }^{54}$. D-gal induced aging mice showed that normal neuronal morphology was damaged, and expression was increased in the hippocampus. GNL, however, restored cell architecture and morphology and reduced neuronal apoptosis. This indicates that GNL protects hippocampal neurons from D-galactose-induced apoptosis. That has been confirmed by histopathological analysis where GNL reduced the neurodegeneration, necrotic injury and decreased numbers of shrunken neurons that were seen in the aging model.

\section{Conclusion}

Results of the current study demonstrate, for the first time, that GNL exhibits a strong antioxidant activity managed to significantly attenuate brain hippocampus injury induced in vivo by D-gal. Through activation of PI3K/Akt/Nrf2 it reduces apoptosis and oxidative stress and then helps in easing cognitive dysfunction and in reducing neurological deficits in D-galactose-induced aging mouse models. According to our study, GNL might be a promising therapeutic agent for age-related diseases.

\section{Declarations}

\section{Funding}

This study supported by Deanship of Scientific Research at King Faisal University, Al Ahsa, Saudi Arabia, and Grant No. AN00084; 2021.

\section{Data Availability Statement}

The data that support the findings of this study are available from the corresponding author upon reasonable request.

\section{Conflicts of Interest}

The authors declare no conflict of interest. 


\section{Contribution}

P.R., E.A.A., R.B.A., F. J.A.S and V.P.V. wrote the main manuscript text. P.R. E.A.A., F.J.A.S., S.Y.R., S.A.A., M.S.M., and N.A. prepared figures and videos P.R and E.A.A acted in a supervisory role. P.R., R.B.A and J.A.S. prepared all figures. All authors reviewed and edited the manuscript.

\section{References}

1. Rehman, S.U., Shah, S.A., Ali, T., Chung, J.I. \& Kim, M.O. Anthocyanins reversed D-galactose-induced oxidative stress and neuroinflammation mediated cognitive impairment in adult rats. Molecular neurobiology 54, 255-271 (2017).

2. Park, M.H., Jin, H.K. \& Bae, J.-s. Potential therapeutic target for aging and age-related neurodegenerative diseases: the role of acid sphingomyelinase. Experimental \& Molecular Medicine, $1-10$ (2020).

3. Xu, L.-Q. et al. Polydatin attenuates d-galactose-induced liver and brain damage through its antioxidative, anti-inflammatory and anti-apoptotic effects in mice. Food \& function 7, 4545-4555 (2016).

4. Oomen, C.A. et al. Resveratrol preserves cerebrovascular density and cognitive function in aging mice. Frontiers in aging neuroscience 1, 4 (2009).

5. Cutler, R.G. et al. Involvement of oxidative stress-induced abnormalities in ceramide and cholesterol metabolism in brain aging and Alzheimer's disease. Proceedings of the National Academy of Sciences 101, 2070-2075 (2004).

6. Ma, J. et al. Combination of chick embryo and nutrient mixture prevent D-galactose-induced cognitive deficits, immune impairment and oxidative stress in aging rat model. Scientific reports 9 , 1-10 (2019).

7. Chen, P., Chen, F. \& Zhou, B. Antioxidative, anti-inflammatory and anti-apoptotic effects of ellagic acid in liver and brain of rats treated by D-galactose. Scientific reports $8,1-10$ (2018).

8. Lu, J. et al. Ursolic acid ameliorates cognition deficits and attenuates oxidative damage in the brain of senescent mice induced by D-galactose. Biochemical pharmacology 74, 1078-1090 (2007).

9. Zhang, Q., Li, X., Cui, X. \& Zuo, P. D-galactose injured neurogenesis in the hippocampus of adult mice. Neurological research 27, 552-556 (2005).

10. Tang, Z. et al. Nrf2 drives oxidative stress-induced autophagy in nucleus pulposus cells via a Keap1/Nrf2/p62 feedback loop to protect intervertebral disc from degeneration. Cell Death \& Disease 10, 1-12 (2019).

11. Cuadrado, A. et al. Therapeutic targeting of the NRF2 and KEAP1 partnership in chronic diseases. Nature Reviews Drug Discovery 18, 295-317 (2019).

12. Rajendran, P., Alzahrani, A.M., Ahmed, E.A. \& Veeraraghavan, V.P. Kirenol Inhibits B [a] P-Induced Oxidative Stress and Apoptosis in Endothelial Cells via Modulation of the Nrf2 Signaling Pathway. 
Oxidative Medicine and Cellular Longevity 2021 (2021).

13. Li, X.-H., Li, C.-Y., Lu, J.-M., Tian, R.-B. \& Wei, J. Allicin ameliorates cognitive deficits ageing-induced learning and memory deficits through enhancing of Nrf2 antioxidant signaling pathways. Neuroscience letters 514, 46-50 (2012).

14. Wang, X.-R. et al. Acupuncture ameliorates cognitive impairment and hippocampus neuronal loss in experimental vascular dementia through Nrf2-mediated antioxidant response. Free Radical Biology and Medicine 89, 1077-1084 (2015).

15. Mao, L. et al. Protective effects of sulforaphane in experimental vascular cognitive impairment: contribution of the Nrf2 pathway. Journal of Cerebral Blood Flow \& Metabolism 39, 352-366 (2019).

16. Gunderson, J.T., Peppriell, A.E., Vorojeikina, D. \& Rand, M.D. Tissue-specific Nrf2 signaling protects against methylmercury toxicity in Drosophila neuromuscular development. Archives of Toxicology 94, 4007-4022 (2020).

17. Zhuang, C.-L. et al. Ginsenoside Rb1 improves postoperative fatigue syndrome by reducing skeletal muscle oxidative stress through activation of the PI3K/Akt/Nrf2 pathway in aged rats. European journal of pharmacology 740, 480-487 (2014).

18. Jeong, H., Liu, Y. \& Kim, H.-S. Dried plum and chokeberry ameliorate d-galactose-induced aging in mice by regulation of Pl3k/Akt-mediated Nrf2 and Nf-kB pathways. Experimental gerontology 95, 16-25 (2017).

19. Zhao, Y., Liu, X., Zheng, Y., Liu, W. \& Ding, C. Aronia melanocarpa polysaccharide ameliorates inflammation and aging in mice by modulating the AMPK/SIRT1/NF-KB signaling pathway and gut microbiota. Scientific reports 11, 1-15 (2021).

20. Lei, Y., Fu, P., Jun, X. \& Cheng, P. Pharmacological properties of geraniol-a review. Planta medica $\mathbf{8 5}$, 48-55 (2019).

21. Qi, F. et al. Geraniol and geranyl acetate induce potent anticancer effects in colon cancer Colo-205 cells by inducing apoptosis, DNA damage and cell cycle arrest. J BUON 23, 346-352 (2018).

22. Wang, J., Su, B., Zhu, H., Chen, C. \& Zhao, G. Protective effect of geraniol inhibits inflammatory response, oxidative stress and apoptosis in traumatic injury of the spinal cord through modulation of NF-кB and p38 MAPK. Experimental and therapeutic medicine 12, 3607-3613 (2016).

23. Pereira, F.d.O. et al. Antifungal activity of geraniol and citronellol, two monoterpenes alcohols, against Trichophyton rubrum involves inhibition of ergosterol biosynthesis. Pharmaceutical biology 53, 228-234 (2015).

24. Chen, P., Chen, F., Lei, J., Li, Q. \& Zhou, B. Activation of the miR-34a-mediated SIRT1/mTOR signaling pathway by urolithin A attenuates D-galactose-induced brain aging in mice. Neurotherapeutics 16, 1269-1282 (2019).

25. Ismail, M.B., Rajendran, P., AbuZahra, H.M. \& Veeraraghavan, V.P. Mangiferin Inhibits Apoptosis in Doxorubicin-Induced Vascular Endothelial Cells via the Nrf2 Signaling Pathway. International Journal of Molecular Sciences 22, 4259 (2021).

26. Sharma, V.K. Morris water maze-a versatile cognitive tool. memory 8 (2009). 
27. Gong, Y.-S. et al. Ameliorative effect of lotus seedpod proanthocyanidins on cognitive impairment and brain aging induced by D-galactose. Experimental gerontology 74, 21-28 (2016).

28. $\mathrm{Yu}, \mathrm{Y}$. et al. Fibroblast growth factor 21 protects mouse brain against D-galactose induced aging via suppression of oxidative stress response and advanced glycation end products formation. Pharmacology biochemistry and behavior 133, 122-131 (2015).

29. Tsai, S.-j. \& Yin, M.-c. Anti-glycative and anti-inflammatory effects of protocatechuic acid in brain of mice treated by D-galactose. Food and chemical toxicology 50, 3198-3205 (2012).

30. Singh, A., Kukreti, R., Saso, L. \& Kukreti, S. Oxidative stress: a key modulator in neurodegenerative diseases. Molecules 24, 1583 (2019).

31. Schmidlin, C.J., Dodson, M.B., Madhavan, L. \& Zhang, D.D. Redox regulation by NRF2 in aging and disease. Free Radical Biology and Medicine 134, 702-707 (2019).

32. Yu, T. et al. Protective effects of selenium-enriched peptides from Cardamine violifolia on dgalactose-induced brain aging by alleviating oxidative stress, neuroinflammation, and neuron apoptosis. Journal of Functional Foods 75, 104277 (2020).

33. Ullah, R. et al. Vanillic Acid, a Bioactive Phenolic Compound, Counteracts LPS-Induced Neurotoxicity by Regulating c-Jun N-Terminal Kinase in Mouse Brain. International Journal of Molecular Sciences 22, 361 (2021).

34. He, L.-L. et al. Qiangji Decoction Alleviates Neurodegenerative Changes and Hippocampal Neuron Apoptosis Induced by D-Galactose via Regulating AMPK/SIRT1/NF-KB Signaling Pathway. Frontiers in Pharmacology, 2104 (2021).

35. Zeng, L., Lin, L., Chen, L., Xiao, W. \& Gong, Z. I-Theanine Ameliorates d-Galactose-Induced Brain Damage in Rats via Inhibiting AGE Formation and Regulating Sirtuin1 and BDNF Signaling Pathways. Oxidative Medicine and Cellular Longevity 2021 (2021).

36. Khaneghah, A.M., Fakhri, Y. \& Sant'Ana, A.S. Impact of unit operations during processing of cerealbased products on the levels of deoxynivalenol, total aflatoxin, ochratoxin $A$, and zearalenone: $A$ systematic review and meta-analysis. Food chemistry 268, 611-624 (2018).

37. M Soliman, S., M Sheta, N., MM Ibrahim, B., M El-Shawwa, M. \& M Abd El-Halim, S. Novel intranasal drug delivery: geraniol charged polymeric mixed micelles for targeting cerebral insult as a result of ischaemia/reperfusion. Pharmaceutics 12, 76 (2020).

38. Farokhcheh, M. et al. Geraniol improved memory impairment and neurotoxicity induced by zinc oxide nanoparticles in male wistar rats through its antioxidant effect. Life Sciences 282, 119823 (2021).

39. Rajendran, P. et al. Antioxidants and human diseases. Clinica chimica acta 436, 332-347 (2014).

40. Rajendran, P. et al. The multifaceted link between inflammation and human diseases. Journal of cellular physiology 233, 6458-6471 (2018).

41. Kaviani, E. et al. Protective effect of atorvastatin on d-galactose-induced aging model in mice. Behavioural brain research 334, 55-60 (2017). 
42. Lin, L. et al. The Inhibitory Efficiencies of Geraniol as an Anti-Inflammatory, Antioxidant, and Antibacterial, Natural Agent Against Methicillin-Resistant Staphylococcus aureus Infection in vivo. Infection and Drug Resistance 14, 2991 (2021).

43. Alzahrani, A.M., Rajendran, P., Veeraraghavan, V.P. \& Hanieh, H. Cardiac Protective Effect of Kirenol against Doxorubicin-Induced Cardiac Hypertrophy in H9c2 Cells through Nrf2 Signaling via PI3K/AKT Pathways. International Journal of Molecular Sciences 22, 3269 (2021).

44. Rajendran, P. et al. Kaempferol inhibits zearalenone-induced oxidative stress and apoptosis via the $\mathrm{PI} 3 \mathrm{~K} /$ Akt-mediated Nrf2 signaling pathway: in vitro and in vivo studies. International Journal of Molecular Sciences 22, 217 (2021).

45. Zhao, M. et al. Bungeanum improves cognitive dysfunction and neurological deficits in D-galactoseinduced aging mice via activating PI3K/Akt/Nrf2 signaling pathway. Frontiers in pharmacology 11, $71(2020)$.

46. Yu, Y. et al. Fibroblast growth factor (FGF21) protects mouse liver against D-galactose-induced oxidative stress and apoptosis via activating Nrf2 and PI3K/Akt pathways. Molecular and cellular biochemistry 403, 287-299 (2015).

47. Jing, L. et al. Structural characterization and antioxidant activity of polysaccharides from Athyrium multidentatum (Doll.) Ching in d-galactose-induced aging mice via PI3K/AKT pathway. Molecules 24, 3364 (2019).

48. Zhang, Y. et al. Naringenin ameliorates behavioral dysfunction and neurological deficits in a dgalactose-induced aging mouse model through activation of PI3K/Akt/Nrf2 pathway. Rejuvenation research 20, 462-472 (2017).

49. Zhao, L. et al. Stevia residue extract ameliorates oxidative stress in d-galactose-induced aging mice via Akt/Nrf2/HO-1 pathway. Journal of Functional Foods 52, 587-595 (2019).

50. Jing, L. et al. Chemical characteristics and cytoprotective activities of polysaccharide fractions from Athyrium Multidentatum (Doll.) Ching. International Journal of Biological Macromolecules 158, 1227-1238 (2020).

51. Song, X. \& Long, D. Nrf2 and ferroptosis: a new research direction for neurodegenerative diseases. Frontiers in neuroscience 14, 267 (2020).

52. Hei, X., Xie, M., Xu, J., Li, J. \& Liu, T. $\beta$-Asarone Exerts Antioxidative Effects on $\mathrm{H} 2 \mathrm{O}$ 2-Stimulated PC12 Cells by Activating Nrf2/HO-1 Pathway. Neurochemical Research 45, 1953-1961 (2020).

53. Zhao, H. et al. Antioxidant effects of compound walnut oil capsule in mice aging model induced by D-galactose. Food \& Nutrition Research 62 (2018).

54. Tsai, S.j. \& Yin, M.-c. Anti-oxidative, anti-glycative and anti-apoptotic effects of oleanolic acid in brain of mice treated by D-galactose. European journal of pharmacology 689, 81-88 (2012).

\section{Figures}


A
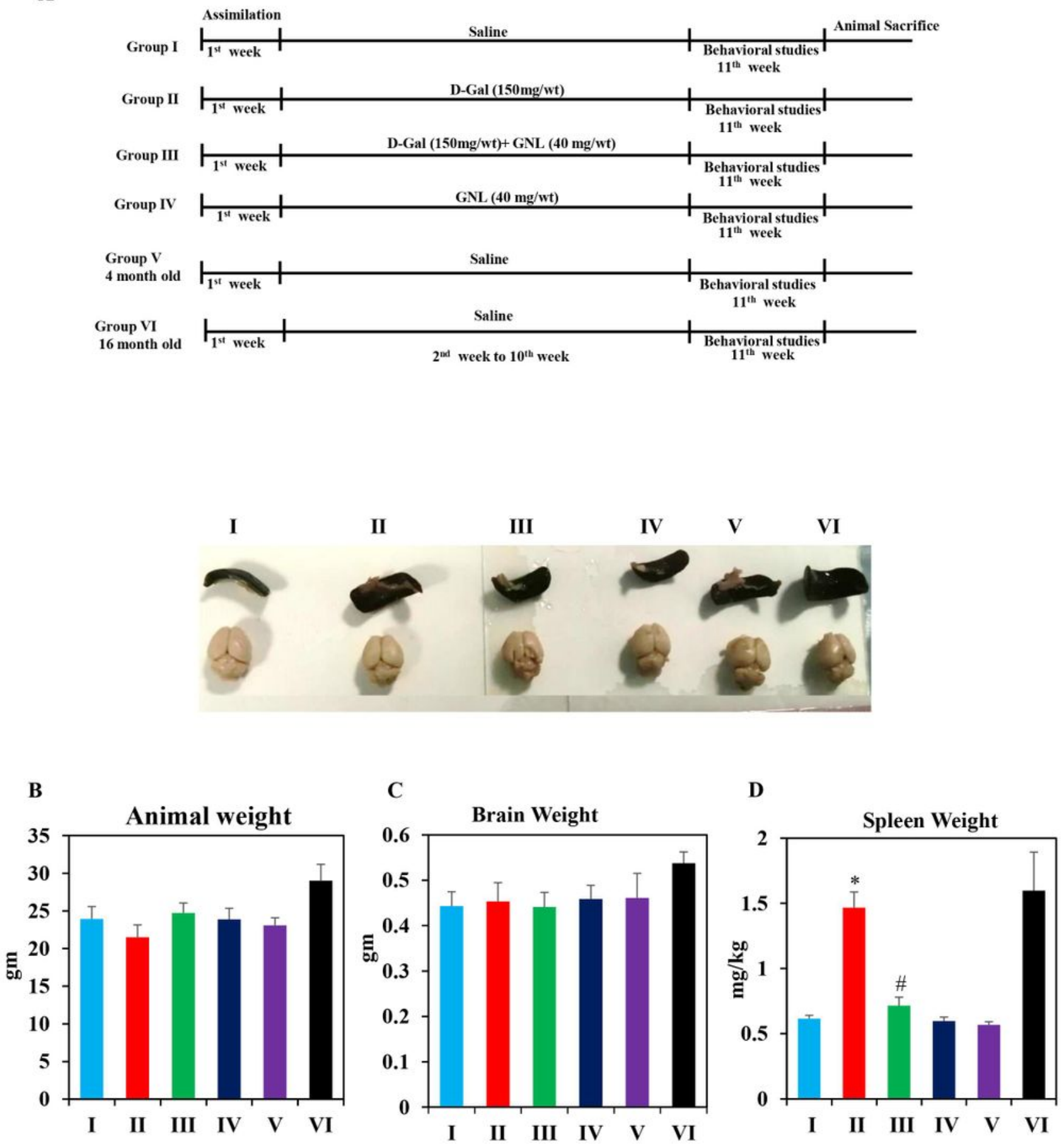

Fig 1

\section{Figure 1}

In D-Gal-induced aging mice, GNL treatment reduces spleen weight. (A), Illustration of the experimental procedure for D-gal and GNL in mice. The first group of animals was given physiological saline solution instead of D-gal (I), and the second group was given D-gal (150 mg/wt) dissolved in normal saline solution $(0.9 \%, \mathrm{w} / \mathrm{v})$ for 9 weeks (II). Animals in the third group of experiments were treated with GNL (40 $\mathrm{mg} / \mathrm{kg} \mathrm{wt}$ ) alone in the first week, and then from the second to the 10th week by oral gavage (without 
anesthesia) with D-gal (150 mg/wt) followed by GNL ( $50 \mathrm{mg} / \mathrm{kg} \mathrm{wt}) 4 \mathrm{~h}$ later (III). Animals in the fourth group were given GNL from the second week until the end of the experiment (IV). We included 6 mice from two untreated groups at 4 months (V) and 6 months (IV) of age for analysis of cognitive impairment. This was done to determine whether treatment prevented or reversed the kinetics of cognitive decline. (B), Body weight (gm), (C), Brain weight (gm) (D), Spleen weight $(\mathrm{mg})$. Data represent mean $\pm S D$. ${ }^{*} P<0.05$ vs control group, $\# \mathrm{P}<0.05$ vs $\mathrm{D}$-gal alone treated mice. ${ }^{@} \mathrm{P}<0.05$ young vs old mice. 

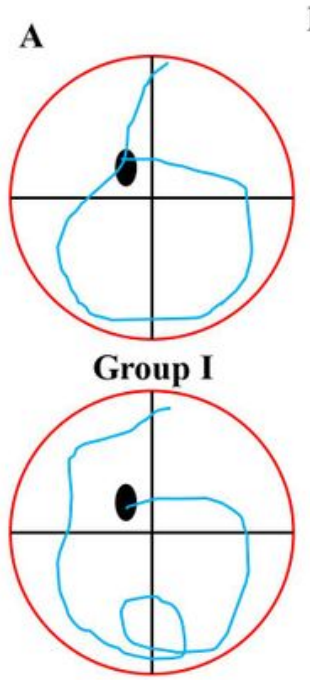

Group IV
Morris Water Maze Test
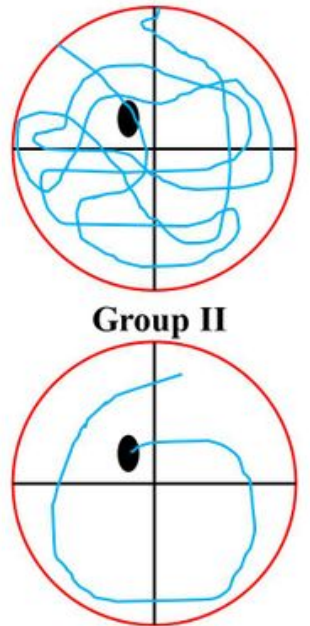

Group V

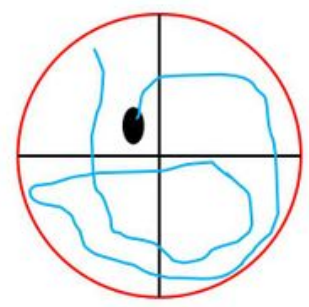

Group III

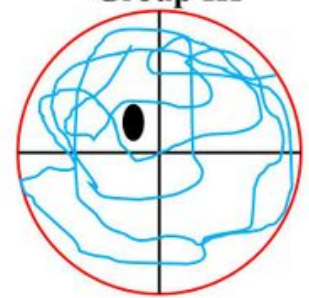

Group VI

B

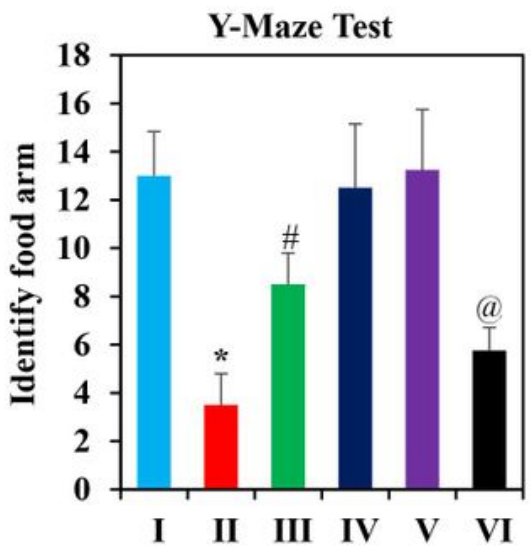

C
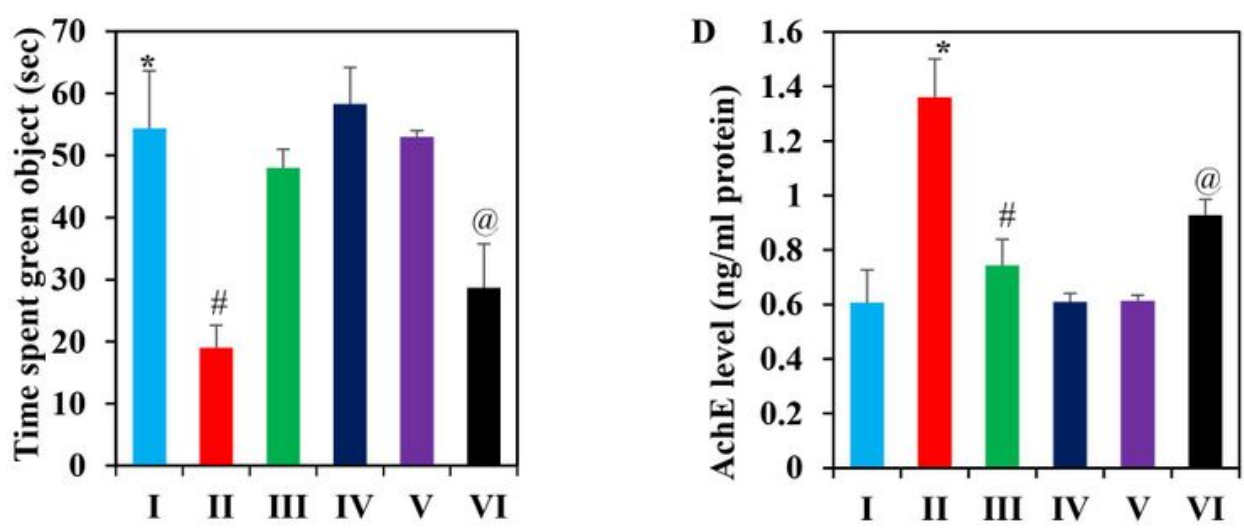

E
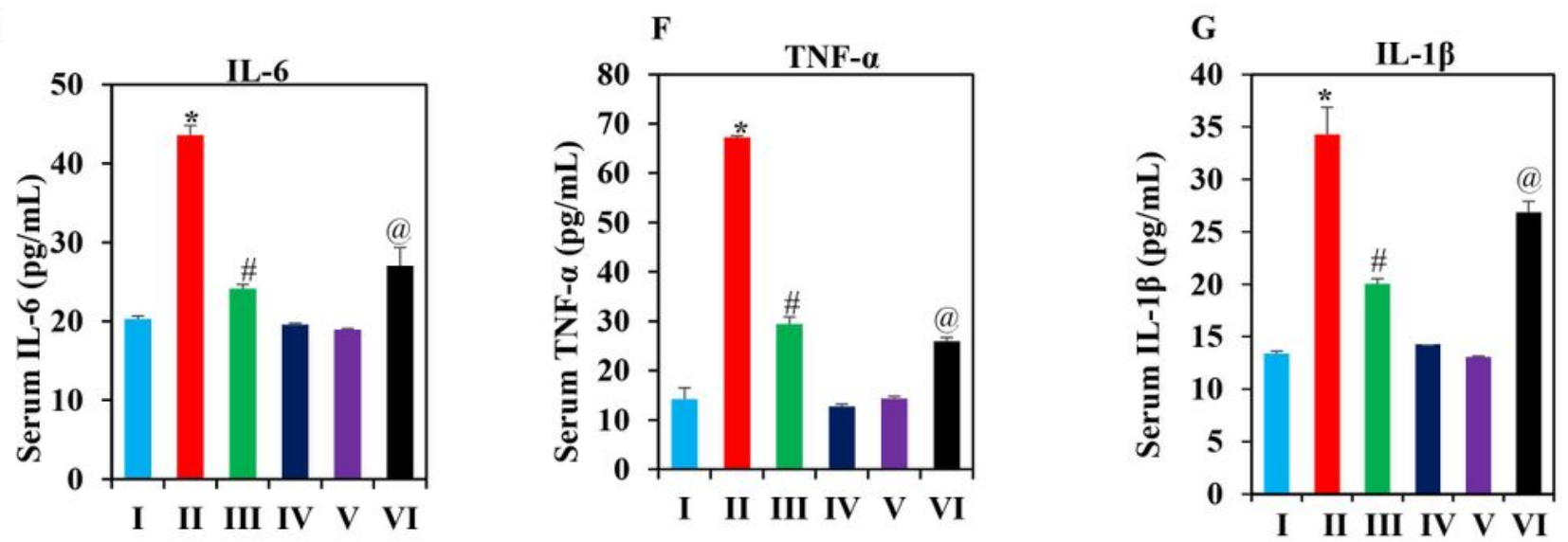

Fig 2

Figure 2

The effects of GNL on cognitive decline induced by D-Gal. (A), Effect of GNL on Mouse roadmap in morris water maze test. (B), effect of GNL on Y-maze task, analyzed for spatial working memoryin D-Gal induced mice. (C), Effect of GNL on object place recognition. (D) Effect of GNL on AchE level in D-Gal induced micev ( $\mathrm{ng} / \mathrm{ml}$ protein). Effect of GNL on inflammatory cytokines in serum such as $(\mathrm{E}) \mathrm{IL}-6(\mathrm{pg} / \mathrm{ml}),(\mathrm{F})$ TNF-a (pg/ml), (G) IL-1 3 (pg/ml). Group I: Control, Group II: D-Gal alone (150mg/wt), Group III: D-Gal 
(150mg/wt) with GNL (40mg/wt), Group IV: GNL alone (40mg/wt). Group V: 4 months young animals; Group Vl: 16 month old. Data represent mean $\pm \mathrm{SD}$. ${ }^{*} \mathrm{P}<0.05$ vs control group, $\# \mathrm{P}<0.05$ vs $\mathrm{D}$-gal alone treated mice. ${ }^{@} \mathrm{P}<0.05$ young vs old mice.

\section{Serum}

A

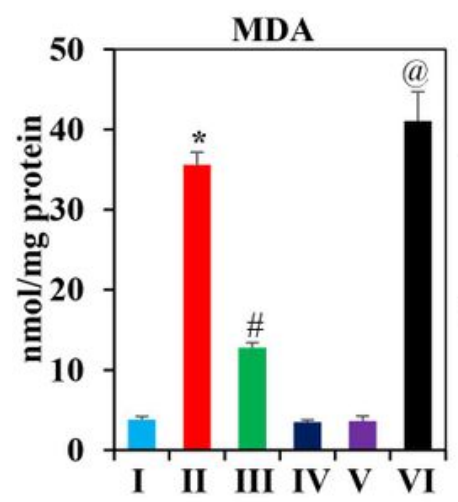

B

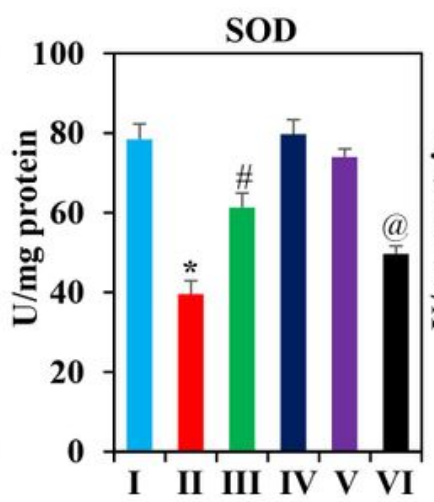

C

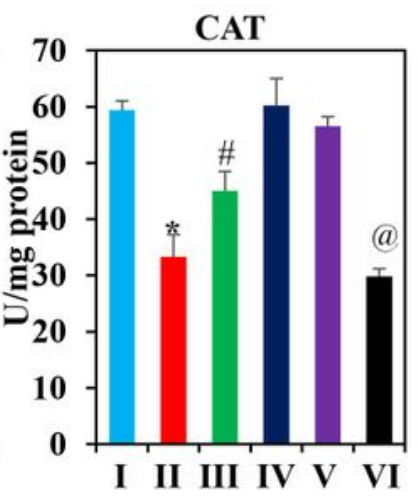

D

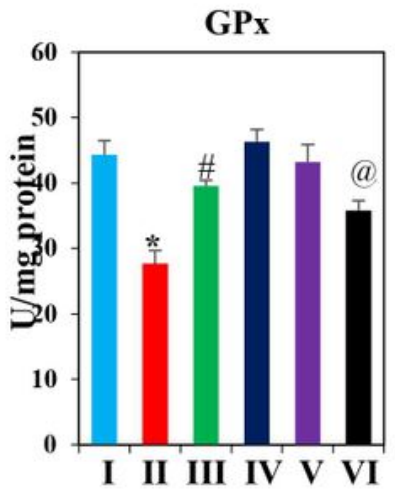

\section{Hippocampus}

E

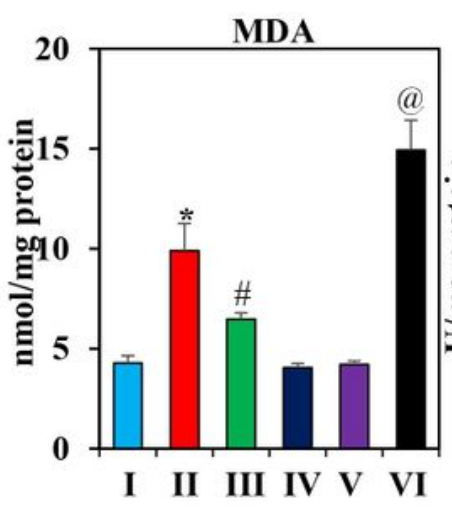

F

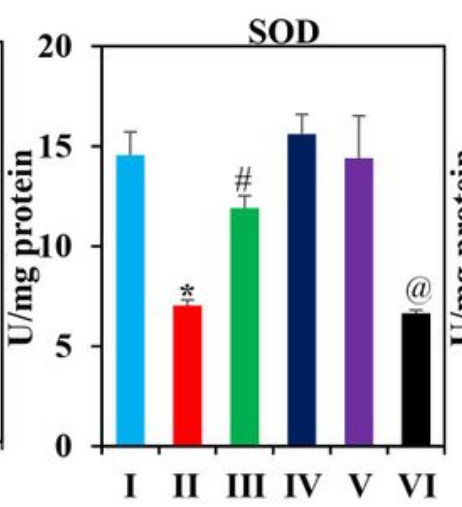

G

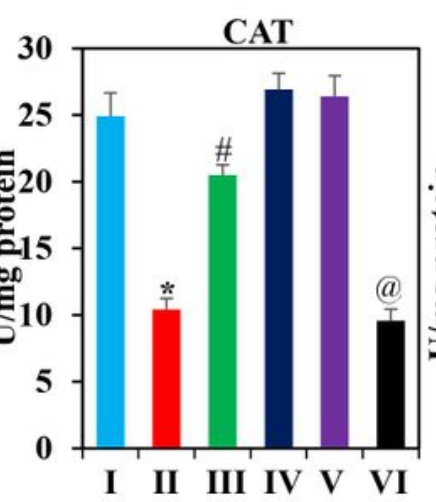

H

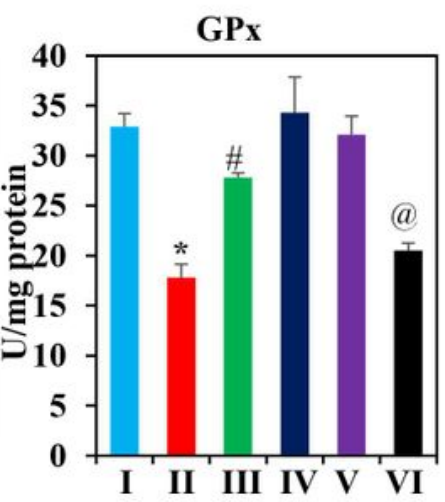

Fig 3

Figure 3 
Effect of GNL on antioxidants activity in D-Gal induced mice of serum and hippocampus. (A) Serum, MDA level control and treated animals (nmol/mg protein), (B) SOD (U/mg protein), (C) CAT (U/mg protein) and (D) GPx (U/mg protein). (E) Hippocampus MDA level control and treated animals (nmol/mg protein), (F) SOD (U/mg protein), (G) CAT (U/mg protein) and (H) GPx (U/mg protein). Data represent mean \pm SD. ${ }^{*} \mathrm{P}<0.05$ vs control group, $\# \mathrm{P}<0.05$ vs $\mathrm{D}$-gal alone treated mice. ${ }^{@} \mathrm{P}<0.05$ young vs old mice.

A
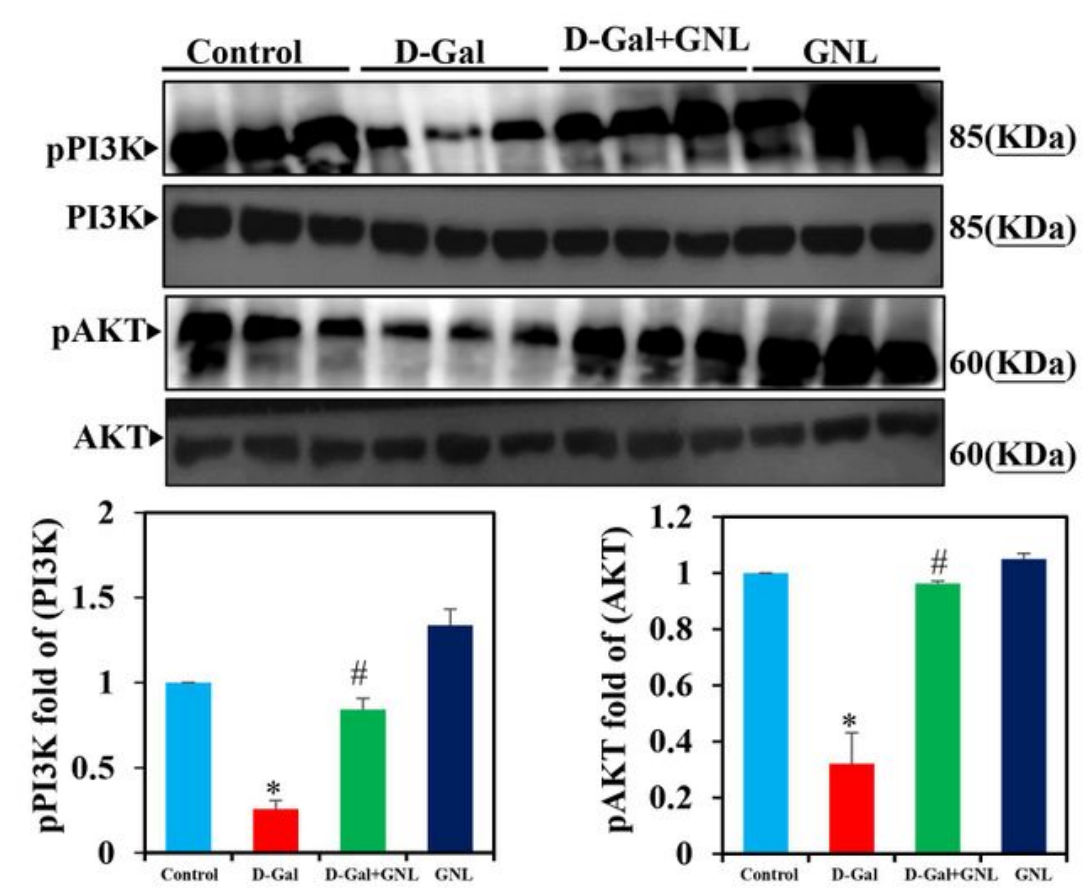

B

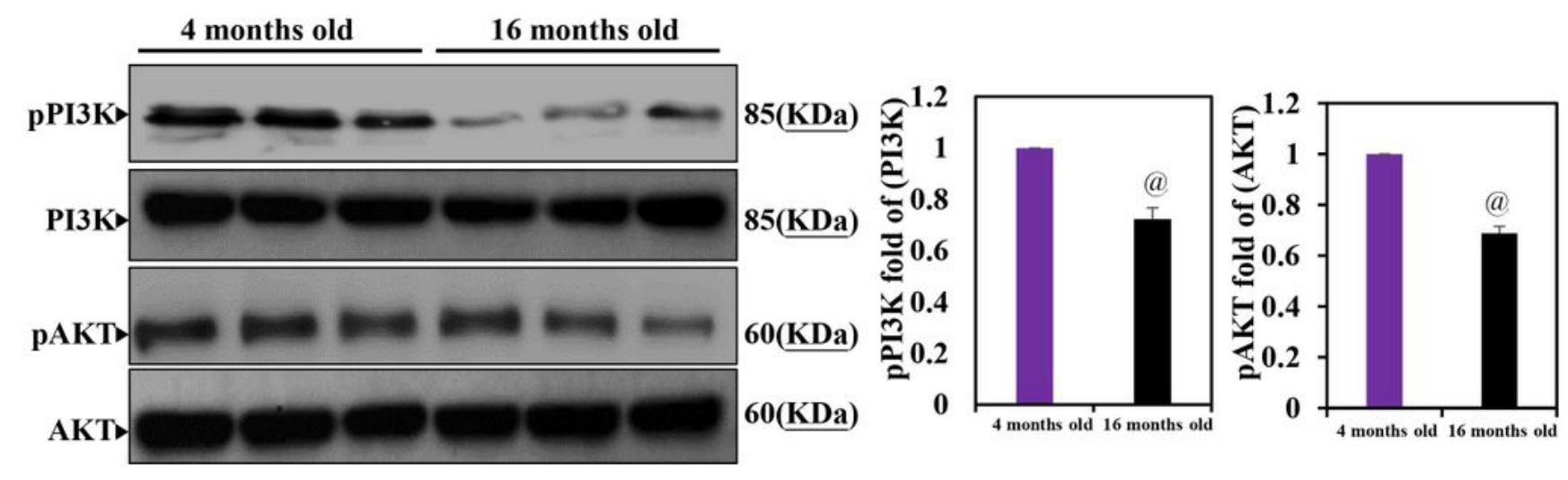

Fig 4 


\section{Figure 4}

GNL affects PI3K/AKT signaling in hippocampus of D-gal-aged mice. (A) pPI3K and pAKT protein analysis by Western blot. Three independent experiments are shown here. SDS-PAGE resolved the protein $(50 \mu \mathrm{g})$ from each sample, and western blots were done. The internal load controllers were AKT and PI3K. Densitometry analysis calculated changes in protein bands as 1.0-fold, as shown below the gel. (B) 4 month young control and 16 months old mice hippocampus tissue analyzed pPI3K and pAKT level. The internal load controllers were AKT and PI3K. Densitometry analysis calculated changes in protein bands as 1.0-fold, as shown right side of the gel. Data represent mean $\pm S D$. ${ }^{*} P<0.05$ vs control group, $\# P<0.05$ vs $\mathrm{D}$-gal alone treated mice. ${ }^{@} \mathrm{P}<0.05$ young vs old mice. 
A
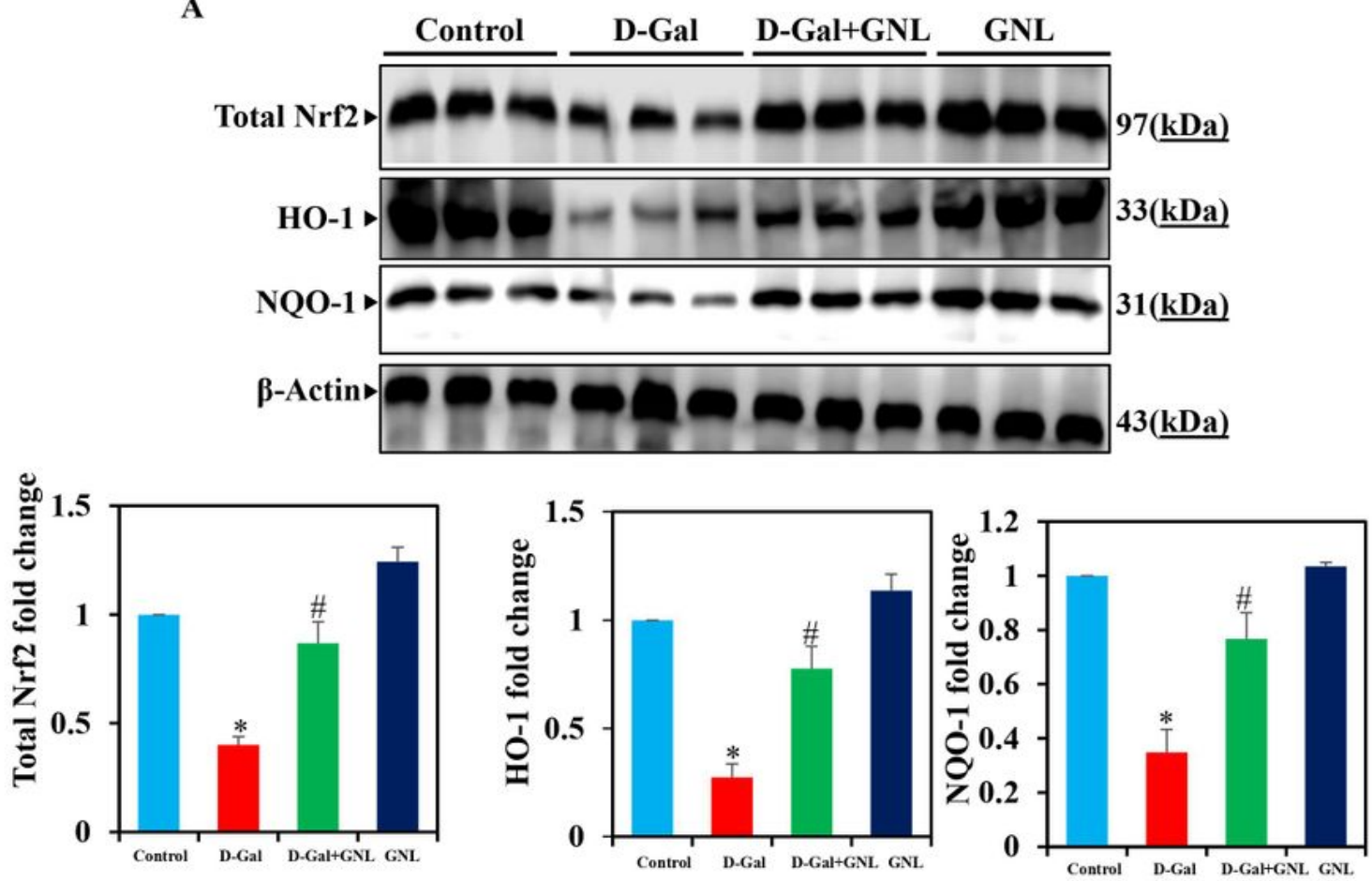

B
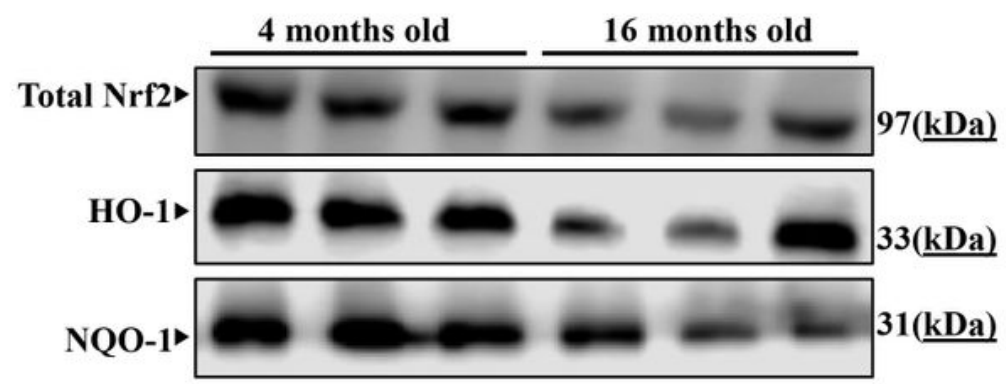

$\beta$-Actin -
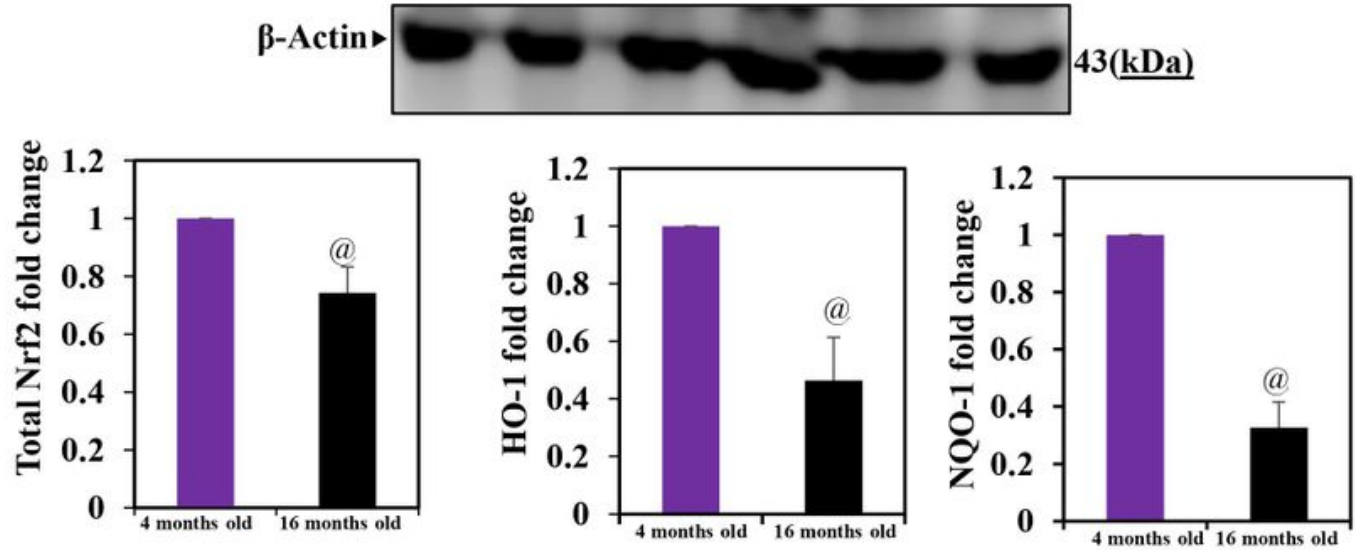

Fig 5

\section{Figure 5}

Effect of GNL on D-Gal induced cognitive impairment mice of Nrf2, HO-1 and NQO-1 proteins in hippocampus. (A) The total Nrf2, HO-1 and NQO-1 protein levels analysis by Western blot. Three independent experiments are shown here. SDS-PAGE resolved the protein $(50 \mu \mathrm{g})$ from each sample, and western blots were done. The internal load controllers were $\beta$-actin. Densitometry analysis calculated changes in protein bands as 1.0-fold, as shown below the gel. (B) 4 month young control and 16 months 
old mice hippocampus tissue analyzed total Nrf2, HO-1 and NQO-1. The internal load controllers were $\beta$ actin. Densitometry analysis calculated changes in protein bands as 1.0 -fold, as shown right side of the gel. Data represent mean \pm SD. ${ }^{*} P<0.05$ vs control group, $\# P<0.05$ vs $D$-gal alone treated mice. ${ }^{@} P<0.05$ young vs old mice.

A
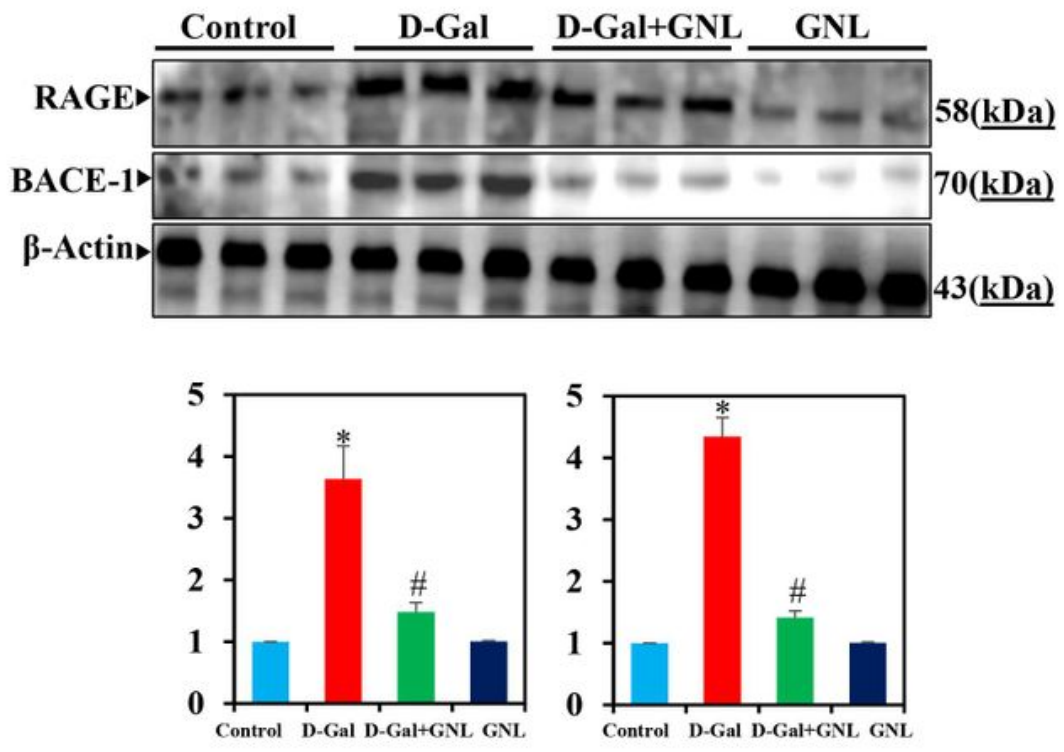

B
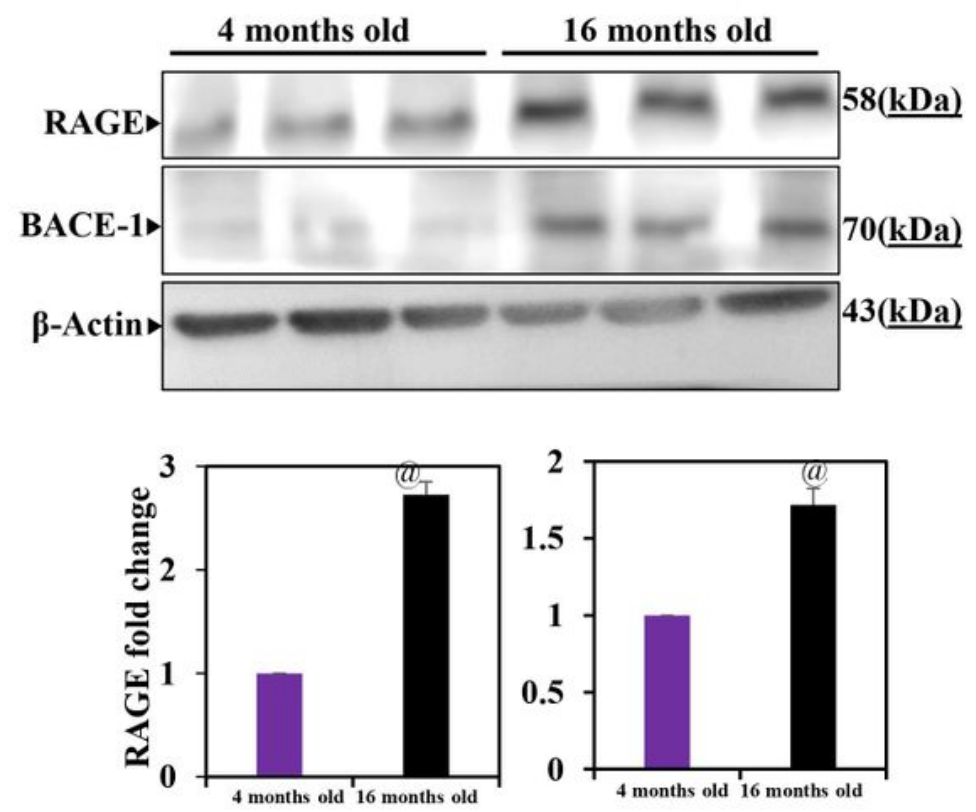

Fig 6 


\section{Figure 6}

Effect of GNL on D-Gal induced cognitive impairment mice of RAGE and BACE-1 proteins in hippocampus. (A) The RAGE and BACE-1 protein levels analysis by Western blot. Three independent experiments are shown here. SDS-PAGE resolved the protein $(50 \mu \mathrm{g})$ from each sample, and western blots were done. The internal load controllers were $\beta$-actin. Densitometry analysis calculated changes in protein bands as 1.0-fold, as shown below the gel. (B) 4 month young control and 16 months old mice hippocampus tissue analyzed RAGE and BACE-1. The internal load controllers were $\beta$-actin. Densitometry analysis calculated changes in protein bands as 1.0-fold, as shown right side of the gel. (C) Hematoxylin staining of the hippocampal region in each group. Group I: Control, Group II: D-Gal alone (150mg/wt), Group III: D-Gal (150mg/wt) with GNL (40mg/wt), Group IV: GNL alone (40mg/wt). Group V: 4 months young animals; Group VI: 16 month old. Data represent mean $\pm S D$. ${ }^{*} \mathrm{P}<0.05$ vs control group, $\# \mathrm{P}<0.05$ vs D-gal alone treated mice. ${ }^{@} \mathrm{P}<0.05$ young vs old mice. 
A
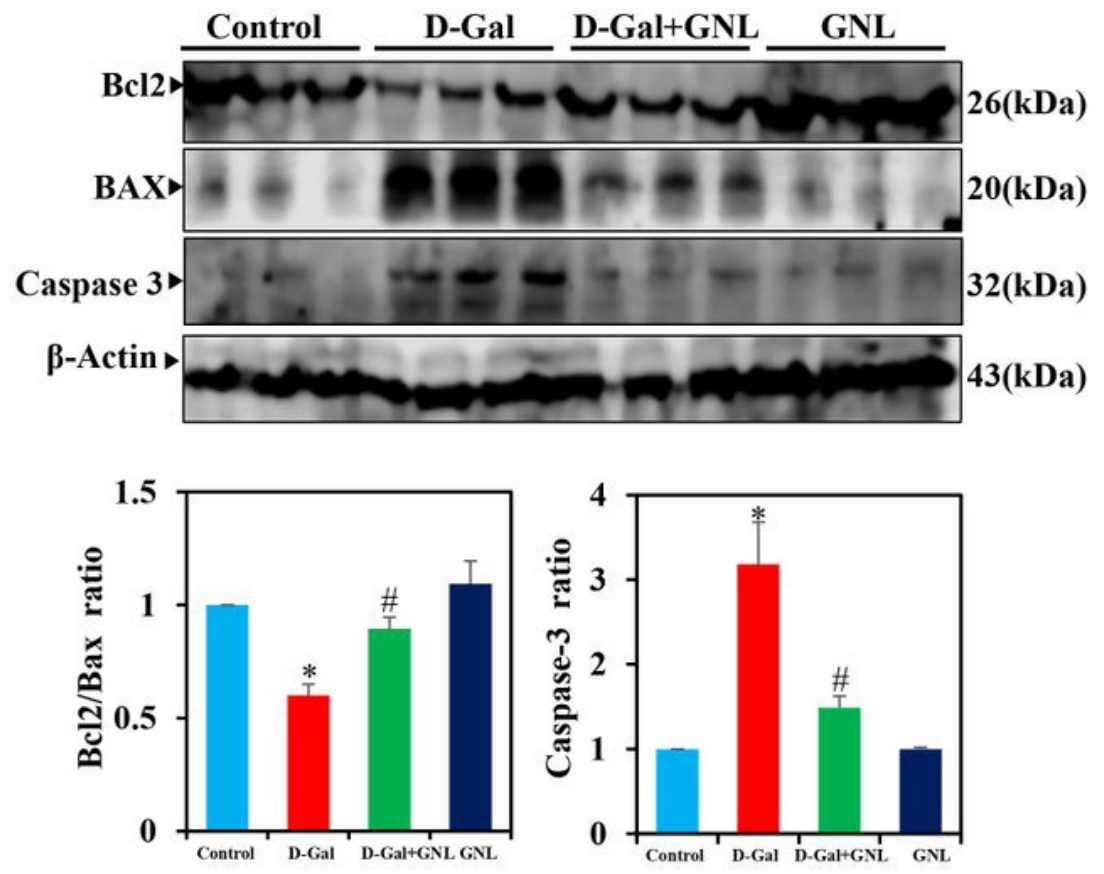

B
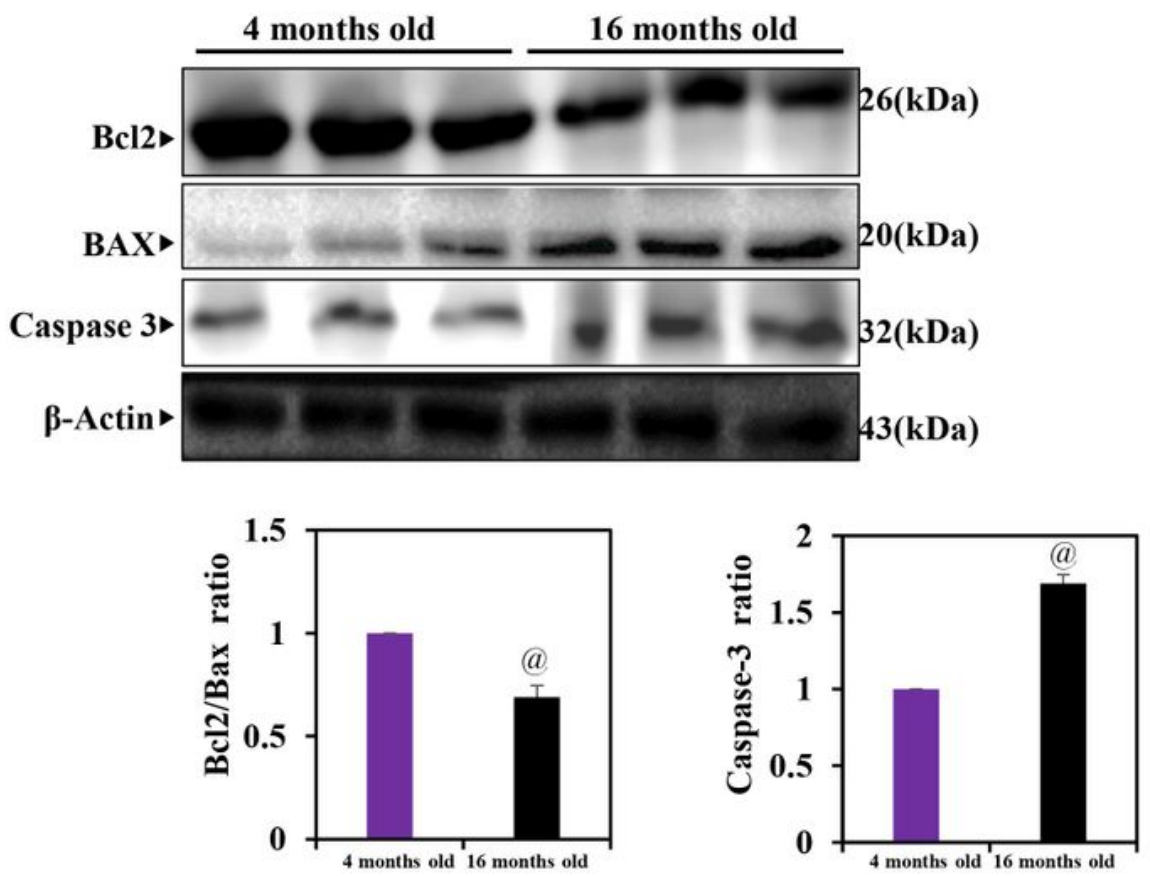

Fig 7

Figure 7

The GNL attenuated excessive apoptosis in mice's hippocampus. (A) The BCl2, BAX and Caspase-3 protein levels analysis by Western blot. Three independent experiments are shown here. SDS-PAGE resolved the protein $(50 \mu \mathrm{g})$ from each sample, and western blots were done. The internal load controllers were $\beta$-actin. Densitometry analysis calculated changes in protein bands as 1.0-fold, as shown below the gel. (B) 4 month young control and 16 months old mice hippocampus tissue analyzed $\mathrm{BCl} 2, \mathrm{BAX}$ and 
Caspase-3. The internal load controllers were $\beta$-actin. Densitometry analysis calculated changes in protein bands as 1.0 -fold, as shown below of the gel. Data represent mean $\pm S D$. ${ }^{*} P<0.05$ vs control group, $\# \mathrm{P}<0.05$ vs $\mathrm{D}-\mathrm{gal}$ alone treated mice. ${ }^{@} \mathrm{P}<0.05$ young vs old mice.
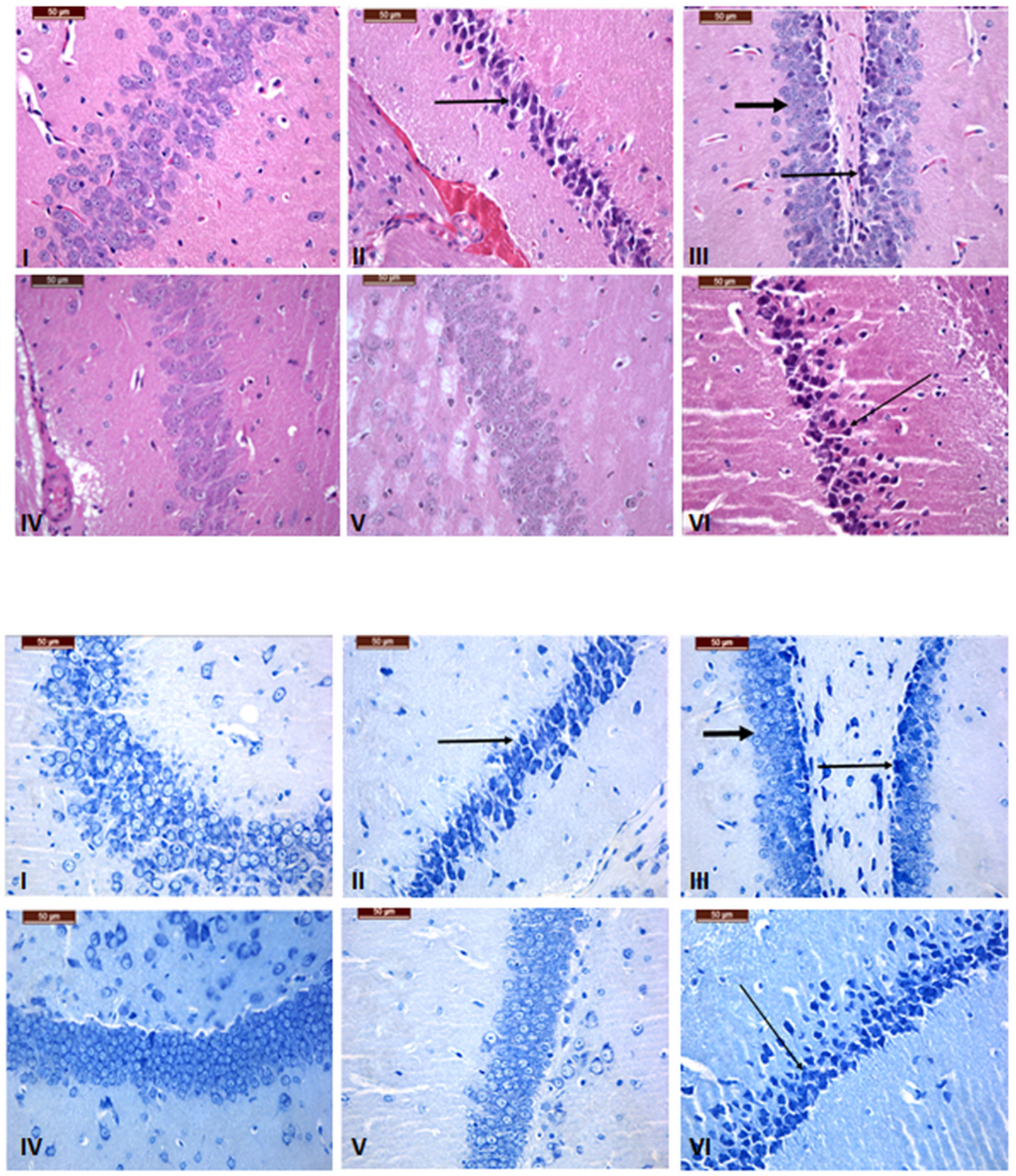

Figure 8 
Histopathological alteration in brain tissue of control and treated groups.

Hippocampus of mice CA1 and CA3 regions stained with H\&E (Left column) and Toluidine blue stain (Right column), (Bar $=100$ and $50 \mathrm{~mm}$ ). G1 (6 weeks), control group, showed normal appearance and high density of neurons with light normal nuclear stain. G2 (6 weeks), Toxic group, showing neurodegeneration characterized by focal disappearance, decrease density, and necrosis represented by shrunken neurons with dark nuclear stained (arrows) compared to the control group. In the G3 (6 weeks), Treatment with Toxin revealed a protective effect of the Drug which showed mild neurodegeneration (thin arrows) with highly pronounced normal neurons (thick arrow). Normal appearance of neurons shown in G4 (6 weeks) Treatment only. In G5 (16 weeks), mild decrease in neuronal density has been shown compared to the control. However, severe neural loss, degeneration, and necrosis (arrows) were seen in old mice G6 (64 weeks).

\section{Supplementary Files}

This is a list of supplementary files associated with this preprint. Click to download.

- Supplementaryfile.pptx

- Videofile1.MorrisWaterMazeTest.pptx

- Videofile2.YMazeTest.pptx

- Videofile3.ObjectPlaceRecognition.pptx 